\title{
It's not always varicocele: A strange case of Zinner syndrome
}

\author{
Nicola Pavan, MD; ${ }^{*}$ Stefano Bucci, MD; Giorgio Mazzon, MD; ${ }^{*}$ Michele Bertolotto, MD; ${ }^{+}$ \\ Carlo Trombetta, MD, ${ }^{*}$ Giovanni Liguori, MD*
}

*Department of Urology, University of Trieste, Trieste, Italy; 'Department of Radiology, University of Trieste, Trieste, Italy

Cite as: Can Urol Assoc J 2015;9(7-8):E535-8. http://dx.doi.org/10.5489/cuaj.2451

Published online July 17, 2015.

\section{Abstract}

A 42-year-old man presented with a huge varicocele. The patient was completely asymptomatic and he did not complain of dysuria, perineal discomfort, or ejaculatory pain. During the visit, the mass mimicked a varicocele and during the Valsalva maneuver a reflux was documented by colour Doppler ultrasound and an abdominal ultrasound revealed an agenesis of the right kidney and a $4.5-\mathrm{cm}$ diameter intra-prostatic cyst. An abdominal and pelvis magnetic resonance imaging was then performed, which confirmed the right renal agenesis, with an epididymal enlargement in the body and tail. This is a unique case of Zinner syndrome in which the patient presented with a paratesticular mass mimicking a varicocele.

\section{Introduction}

We present a case of Zinner syndrome in a patient with a history of painless right scrotal lump. This is a unique case of Zinner syndrome in which the patient presented with a paratesticular mass mimicking a varicocele.

\section{Case report}

A 42-year-old man referred presented with a 4-month history of painless right scrotal lump. He denied lower urinary tract symptoms, gross hematuria, penile discharge, fever, and trauma. Two years prior he had the same problem which resolved with antibiotics and non-steroidal anti-inflammatory medications.

He fathered two children. His physical examination, in the upright position, revealed a mass of tortuous structures like a "bag of worms" in the posterosuperior part of the right scrotum. The right testicle was normal. The texture decreased while he was in the supine position and was exacerbated by the Valsalva maneuver.
The patient underwent a scrotal ultrasound in the emergency room. The testicles presented bilateral normal volume $(15 \mathrm{~mL})$, as well as normal homogeneous echo texture, without calcifications or dilation of the rete testis. Multiple right paratesticular masses were dilated and convoluted structures were present, which appeared irregularly dilated up until the internal inguinal ring. During the Valsalva maneuver, a reflux was documented by colour Doppler ultrasound (Fig. 1). These findings were consistent with the diagnosis of varicocele.

A second-look scrotal colour Doppler ultrasound with a high frequency probe was then performed in our department by a urologist with specific expertise in scrotal ultrasound. The examination confirmed the presence of an abnormal dilatation and tortuosity of the paratesticular structures in the spermatic cord (more than $2 \mathrm{~mm}$ in diameter) with reflux during the Valsalva maneuver, which was still believed to be consistent with a right varicocele. However, three factors emerged during the gray-scale evaluation: (1) the paratesticular palpable mass had an "honeycomb" appearance; (2) the convoluted structures had thickened walls with increased echogenicity (Fig. 2); and (3) it was impossible to identify the body and the tail of epididymis. We decided to perform an abdominal ultrasound, which revealed an agenesis of the right kidney, and a 4.5-cm diameter intra-prostatic cyst (Fig. 3).

Abdominal and pelvis magnetic resonance imaging was then performed, which confirmed a right renal agenesis. Furthermore, epididymal enlargement in the body and tail was evident. The vas deferens was also dilated in all of its length, up until the most cranial portion, where it was absent for $3 \mathrm{~cm}$. It was again irregularly dilated in the pre-terminal portion, leading to a large cyst in the right seminal vesicle $(4.2 \mathrm{~cm}$ in diameter) (Fig. 4).

In view of the above-mentioned clinical and radiological findings, the diagnosis of varicocele was incorrect and Zinner syndrome was diagnosed. The patient was completely asymptomatic and did not complain of dysuria, perineal discomfort, or ejaculatory pain. We kept him in a follow-up program; at the 6-month follow-up, he remained asymptomatic. 


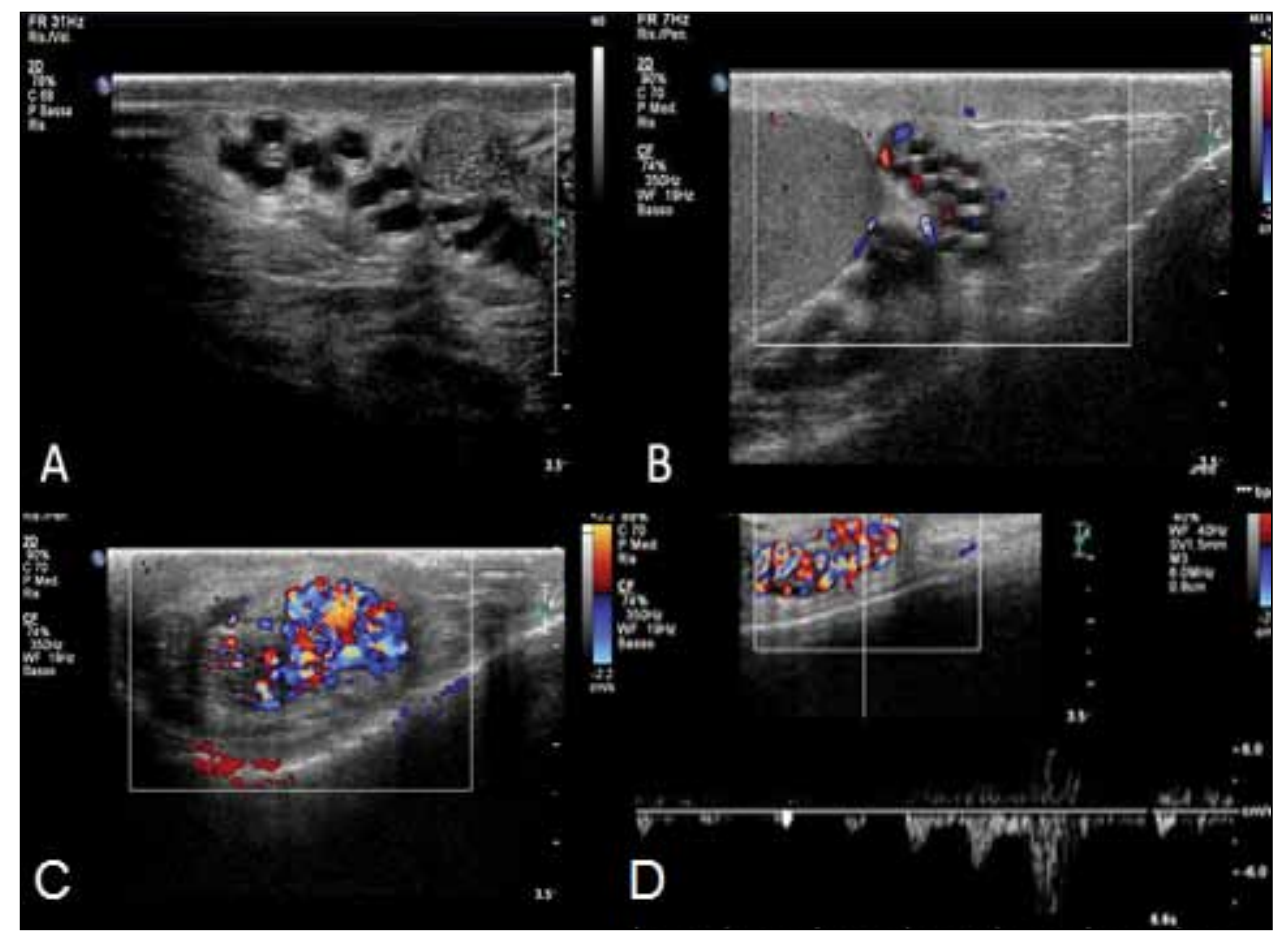

Fig. 1. Grey-scale ultrasound identifies multiple dilated ducts (A). Colour Doppler ultrasound at rest highlights the lack of flow within the ducts (B). Colour Doppler ultrasound shows a retrograde flow lasting more than 2 seconds during Valsalva (C, D).

\section{Discussion}

Zinner syndrome is a triad of mesonephric duct abnormality comprising of unilateral renal agenesis, ipsilateral seminal vesicle cyst, and ejaculatory duct obstruction. Zinner syndrome is related with an embryologic anomaly development between the 4th and the 13th gestational week of the distal portion of the mesonephric or Wolffian duct. ${ }^{1,2}$ Incomplete migration of the ureteric bud, which also arises from the mesonephric duct, may also occur, resulting in a failure to fuse with the metanephros. Presumably, the maldevelopment of the distal mesonephric duct and faulty ureteral budding leads to renal agenesis or dysplasia, and atresia of the ejaculatory duct leads to obstruction and cystic dilatation of the seminal vesicle.

Patients are generally diagnosed during the third or fourth decade, at the time of greatest sexual activity, when the cyst enlarges sufficiently to produce symptoms. ${ }^{3}$ Although

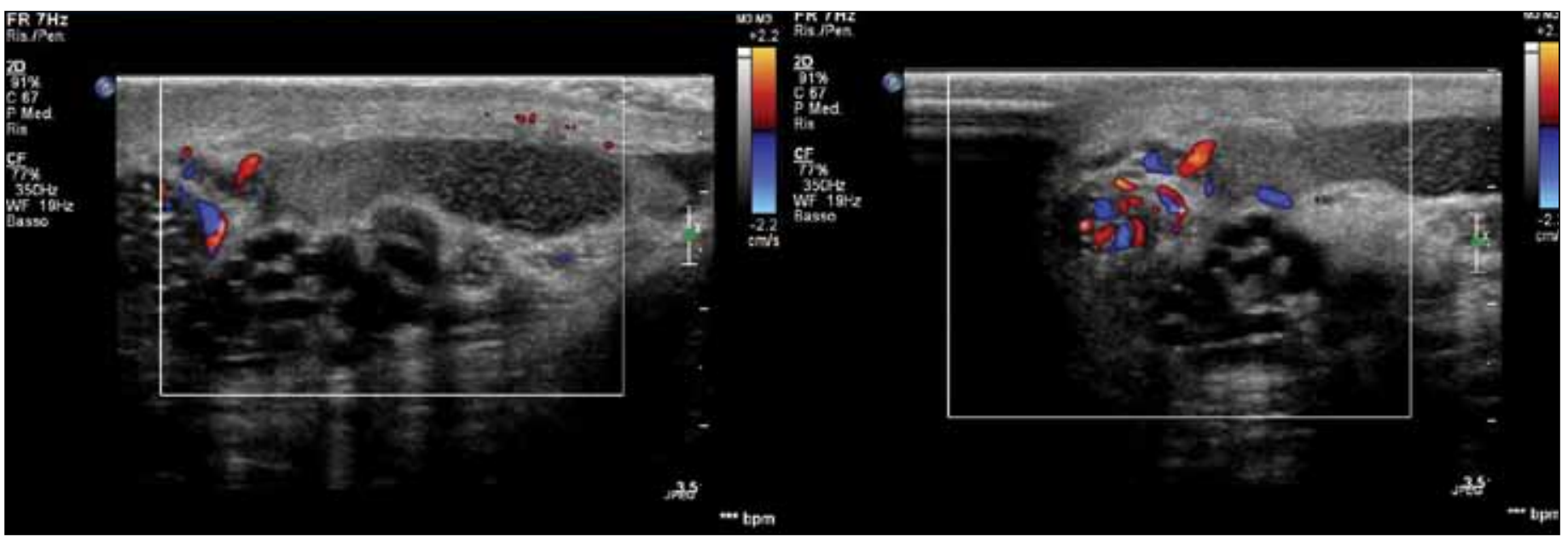

Fig. 2. "Honeycomb" appearance of the paratesticular palpable mass (A); the convoluted structures had thickened walls with increased echogenicity (B). 


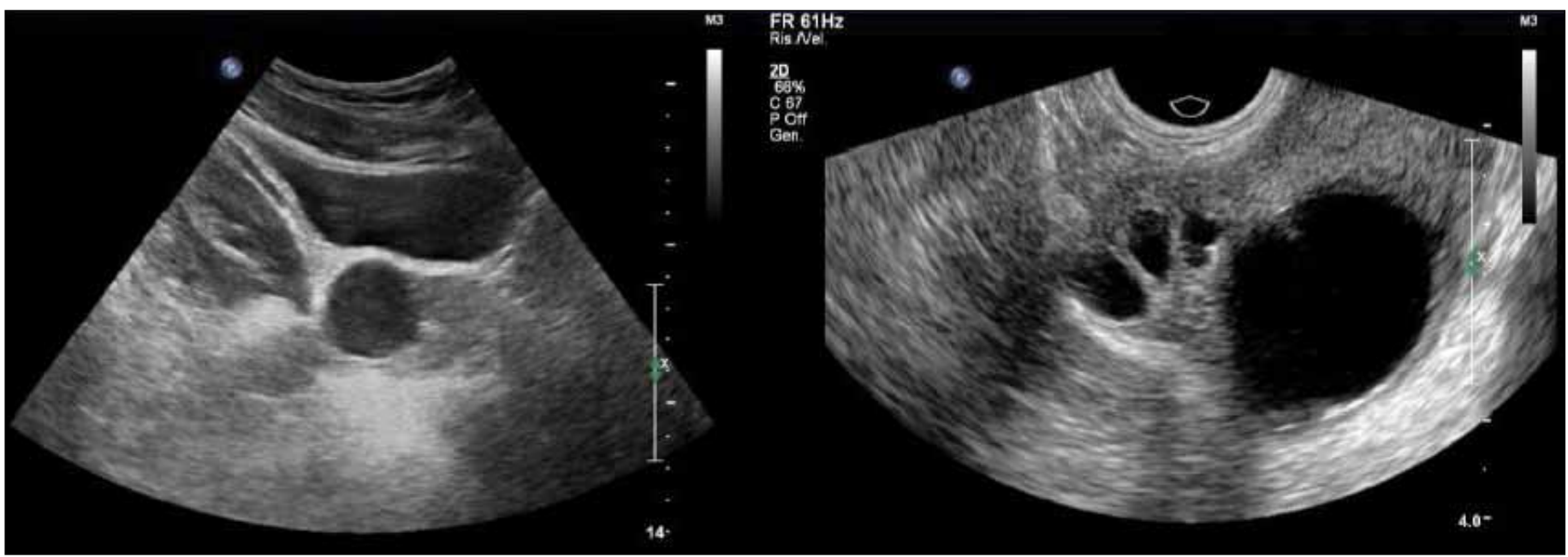

Fig. 3. Suprapubic and transrectal ultrasound. The images show a large cyst of the prostate.

most patients are asymptomatic and diagnosed occasionally, some patients may present with pelvic pain, perineal discomfort, painful ejaculation, or lower urinary tract symptoms. ${ }^{4}$ Occasionally, infertility may be the chief complaint. Seminal vesicle cysts smaller than $5 \mathrm{~cm}$ in diameter are usually asymptomatic; on the contrary, once seminal vesicle cysts exceed $5 \mathrm{~cm}$, the clinical symptoms become evident. Our case uniquely illustrates a paratesticular mass mimicking a varicocele.

A number of imaging modalities, such as seminal vesciculography, transrectal and abdominal ultrasound, and computed tomography, have been used to evaluate patients and have been well-described in the literature. More recently, magnetic resonance imaging has been very accurate in the differential diagnosis of seminal vesicle cysts, ${ }^{5}$ without needing to resort to more invasive investigations. ${ }^{6,7}$

In this case our diagnostic flowchart was misinformed by clinical presentation, physical examination, and by the ultrasound. In particular the ultrasonic appearance of paratesticular hypo-anechoic dilated and convoluted structures with reflux during Valsalva maneuver led us to an initial incorrect diagnosis of varicocele.

Invasive treatment should be restricted to symptomatic cases or to patients who failed conservative management. Treatment usually includes seminal way disclosure via transurethral resection of the ejaculatory duct and open, laparoscopic or robotic vesiculectomy. ${ }^{3}$ Transrectal or transperineal aspiration is another option to relieve symptoms and to establish the diagnosis by allowing instillation of contrast material, ${ }^{7}$ but simple cyst drainage is associated with the risk of recurrence, the return of symptoms, and possible infection. Open excision requires extensive dissection due to the deep location of seminal vesicles, and is associated with a high rate of morbidity. ${ }^{8}$ For this reason, laparoscopy has been advocated as the best minimally invasive technique to surgically treat seminal vesicle pathology. ${ }^{9}$ Robotic- assisted excision of the seminal vesicle cyst using the da Vinci Robotic Surgical System (Intuitive Surgical Systems, Inc.) has also been described with good results. ${ }^{10}$ In our case the patient was completely asymptomatic and preferred a conservative option. He is currently being followed and remains asymptomatic.

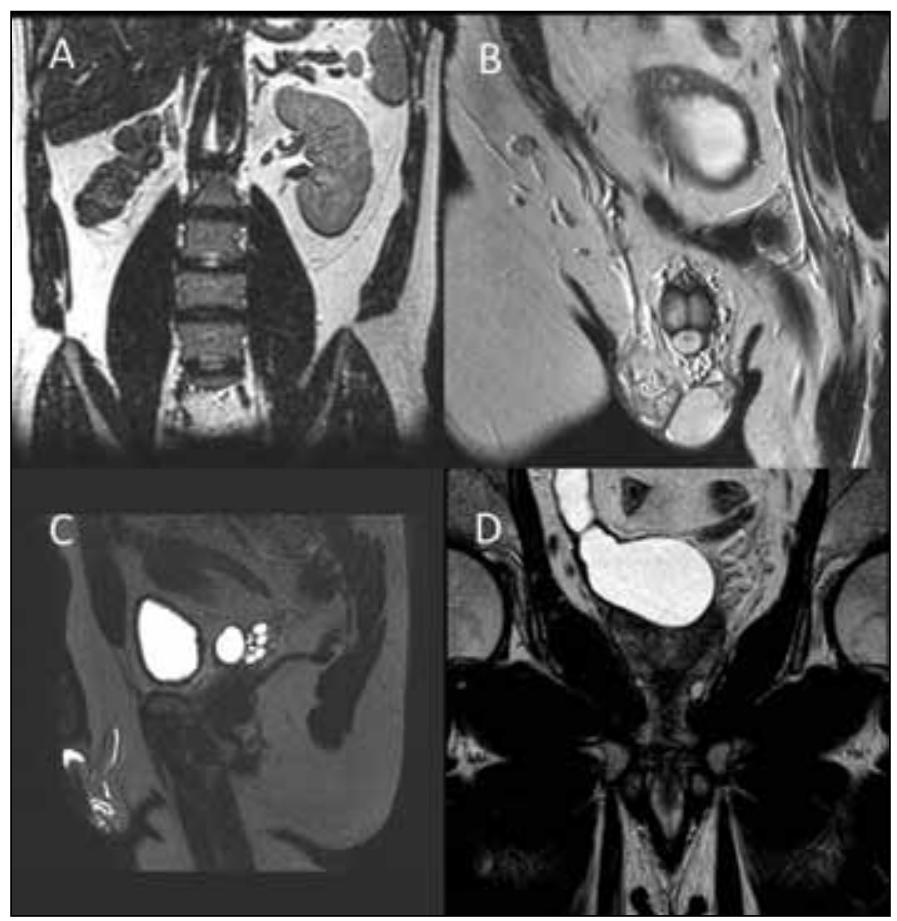

Fig. 4. Magnetic resonance scan of the abdomen and pelvis with frontal slice showing the agenesis of the right kidney (A). The dilation of seminal ducts are well shown in frontal (B) and sagittal slices (C). The cyst of the right seminal vesicle, from which comes the dilated right vas deferens $(C, D)$. 
Pavan et al.

\section{Conclusion}

Our case of an adult male with asymptomatic unilateral palpable mass on the scrotum suggested a diagnosis of varicocele. In the end, the patient had an obstruction to seminal ducts with a cyst in the seminal vesicle and unilateral renal agenesis. To the best of our knowledge, the association between reflux at colour Doppler examination and obstruction of the vas deferens has never before been reported.

Competing interests: The authors declare no competing financial or personal interests.

This paper has been peer-reviewed.

\section{References}

1. Patel B, Guiral S, Jefferson K, et al. Seminal vesicle cysts and associated anomalies. BJU Int 2002;90:26571. http://dx.doi.org/10.1046/i.1464-410X.2002.02883.x

2. Pace G, Galatioto GP, Gualà L, et al. Ejaculatory duct obstruction caused by a right giant seminal vesicle with an ipsilateral upper urinary tract agenesia: An embryologic malformation. Fertil Steril 2008;89:390-4. http://dx.doi.org/10.1016/i.fertnstert.2007.03.009
3. Pereira BJ, Sousa L, Azinhais $P$, et al. Zinner's syndrome: An up-to-date review of the literature based on a clinical case. Andrologia 2009;41:322-30. http://dx.doi.org/10.1111/j.1439-0272.2009.00939.x

4. Ghonge NP, Aggarwal B, Sahu AK. Zinner syndrome: A unique triad of mesonephric duct abnormalities as an unusual cause of urinary symptoms in late adolescence. Indian J Urol 2010;26:444-7. hittp:// dx.doi.org/10.4103/0970-1591.70592

5. Casey RG, Stunell H, Buckley 0 , et al. A unique radiological pentad of mesonephric duct abnormalities in a young man presenting with testicular swelling. Br J Radiol 2008;81:e93-6. http://dx.doi. org $/ 10.1259 / \mathrm{bir} / 31182823$

6. Gozen AS, Alagol B. Endoscopic management of seminal-vesical cyst with right renal agenesis causing acute urinary retention: Case report. J Endourol 2006;20:919-22. http://dx.doi.org/10.1089/ end.2006.20.919

7. Narlawar RS, Hanchate V, Raut A, et al. Renal genesi and seminal vesicle cyst. J Ultrasound Med 2003;22:225-8.

8. Manousakas T, Kyriakou G, Serafetinides E, et al. Partial vesiculectomy in an infertile man with seminal vesicle cyst, ipsilateral renal agenesis, and cryptorchidism. Urology 2002;59:602. http://dx.doi. org/10.1016/50090-4295(01)01675-2

9. Cherullo EE, Meraney AM, Bernstein LH, et al. Laparoscopic management of congenital seminal vesicle cysts associated with ipsilateral renal agenesis. J Urol 2002;167:1263-67. http://dx.doi.org/10.1016/ S0022-5347(05)65278-0

10. Carmack AJ, Siddiq FM, Leveillee RJ. Novel use of da Vinci Robotic Surgical System: Removal of seminal vesicle cyst in previously dissected pelvis. Urology 2006;67:199. http://dx.doi.org/10.1016/i.urology.2005.07.023

Correspondence: Dr. Nicola Pavan, Department of Urology, Cattinara Hospital, Strada di Fiume 44734149 Trieste; nicpavan@gmail.com 